\title{
Statistics of Particle Velocities in Dense Granular Flows
}

\author{
Sudheshna Moka* and Prabhu R. Nott \\ Department of Chemical Engineering, Indian Institute of Science, Bangalore 560 012, India
}

\begin{abstract}
We present measurements of the particle velocity distribution in the slow flow of granular material through vertical channels. The velocities of particles adjacent to the smooth, transparent front face of the channel were determined by video imaging and particle tracking. We find that the mean velocity changes sharply in shear layers near the side walls, but remains constant in a substantial core. The velocity distribution is non-Gaussian, is anisotropic, and follows a power law at large velocities. Remarkably, the distribution is identical in the shear layer and the core. We show evidence of spatially correlated motion, and propose a mechanism for the generation of fluctuational motion in the absence of shear.
\end{abstract}

Granular flows are ubiquitous in nature (rock and snow avalanches, movement of sand dunes) and in industrial processes (transport of food grains, ores, pharmaceutical powders), yet they are poorly understood. In the regime of dense, slow flow there is enduring contact between particles, and shear stresses are generated primarily by dry friction [1,2]. In this regime, it is often observed [3-5] that there is fluidlike deformation only in thin layers, while large regions resist continuous deformation despite the action of a finite shear stress, like a solid. As in molecular fluids, knowledge of the statistics of particle motion will enhance our understanding of the hydrodynamics of granular materials and provide insight into the microscopic basis of the fluidlike and solidlike responses. Moreover, the marked difference in the way dense granular materials and conventional fluids respond to applied forces prompts the question of whether there is a fundamental difference in their statistical nature.

Experimental investigations of the statistics of slow granular flows have been hampered by difficulties in probing dense and opaque systems. Studies on two-dimensional layers of photoelastic disks [6,7] have revealed an anisotropic and inhomogeneous network of "force chains." The contact force distribution is found to decay exponentially for large forces [8], which has been corroborated by particle dynamics simulations [9-11]. Measurements of particle displacements by an indirect, spatially averaged technique [12] and by direct particle tracking near a transparent wall [13] show diffusive motion at long time scales, but the studies differ on their inferences of the microscopic mechanism at play - the former concludes that diffusion arises after repeated impulsive collisions between particles, but the latter attributes it to a mechanism of rearrangement in the network of abiding grain contacts.

In our investigation, the flow of dry, cohesionless, spherical, almost monodisperse glass beads [Fig. 1(b)] of mean diameter $d_{p}=0.8 \mathrm{~mm}$ through a vertical channel was studied. A hopper fed the glass beads into the channel, and the flow rate was controlled by the size of the exit slot at the base of the channel [Fig. 1(a)]. The channel width
$2 W$ and the size of the exit slot could be easily varied; here we present data for $W$ in the range $23 d_{p}-64 d_{p}$. The front and back faces of the channel were smooth, transparent glass plates. The aluminum side walls were roughened by sticking 80 grit sandpaper on them, but were left uncoated in some experiments to study the effect of wall roughness.
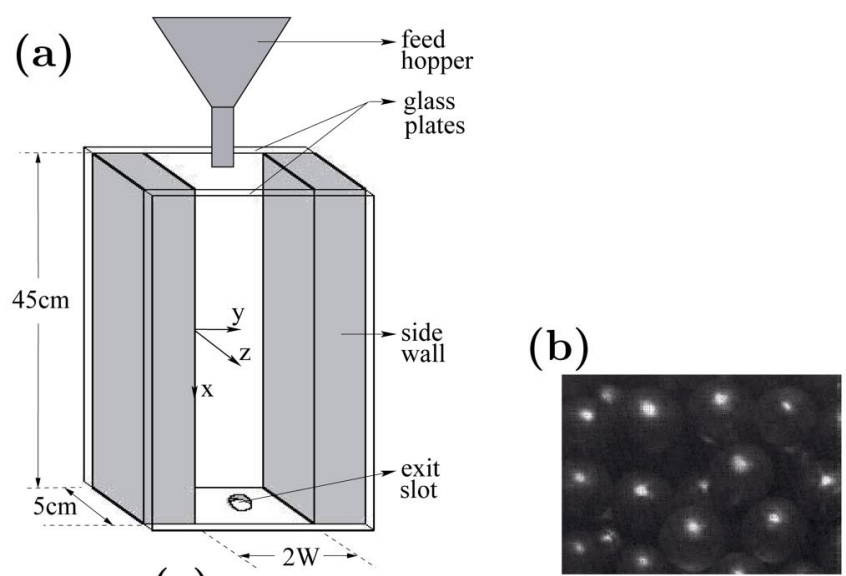

(c)

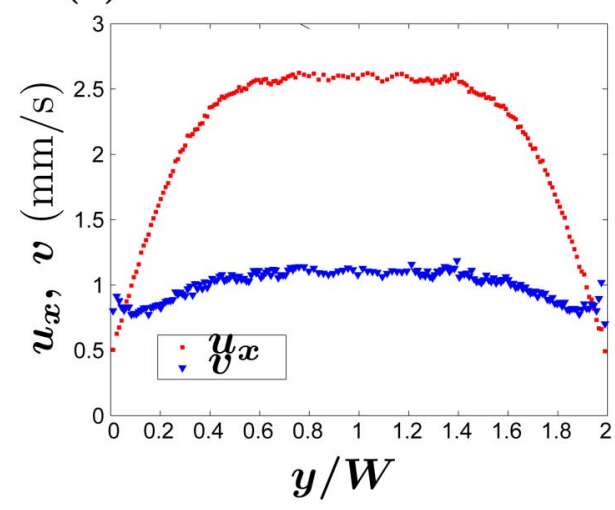

FIG. 1 (color online). (a) Sketch of the experimental assembly (not to scale). (b) Snapshot of the flowing glass beads, showing the bright spots of reflected light. (c) Profiles of the mean velocity $u_{x}$ and the root-mean-square velocity fluctuation $v$ for a rough walled channel with $W / d_{p}=31.7$. 
On illuminating the channel from the front, the reflected light from the beads close to the front glass plate were visible as bright spots [Fig. 1(b)]. Video images of the flowing beads were taken with a CCD camera (mounted on a translating stage) at 25 frames/s, for at least six minutes at each location in the channel, and transferred simultaneously to a computer. Visual inspection of the video movies clearly revealed long-term contact between particles, with rubbing and rolling interactions predominating. Each video frame was analyzed, using standard image analysis techniques, to determine the centroids of the bright spots [Fig. 1(b)]. The velocity components $c_{x}$ and $c_{y}$ of the particles were determined from the displacement of the centroids between adjacent frames. To ensure that spots in adjacent frames were from the same particle, the velocity of a particle was determined only if its displacement was less than $d_{p} / 2$, limiting the maximum measurable velocity to roughly $10 \mathrm{~mm} / \mathrm{s}$. All measure-

(a)
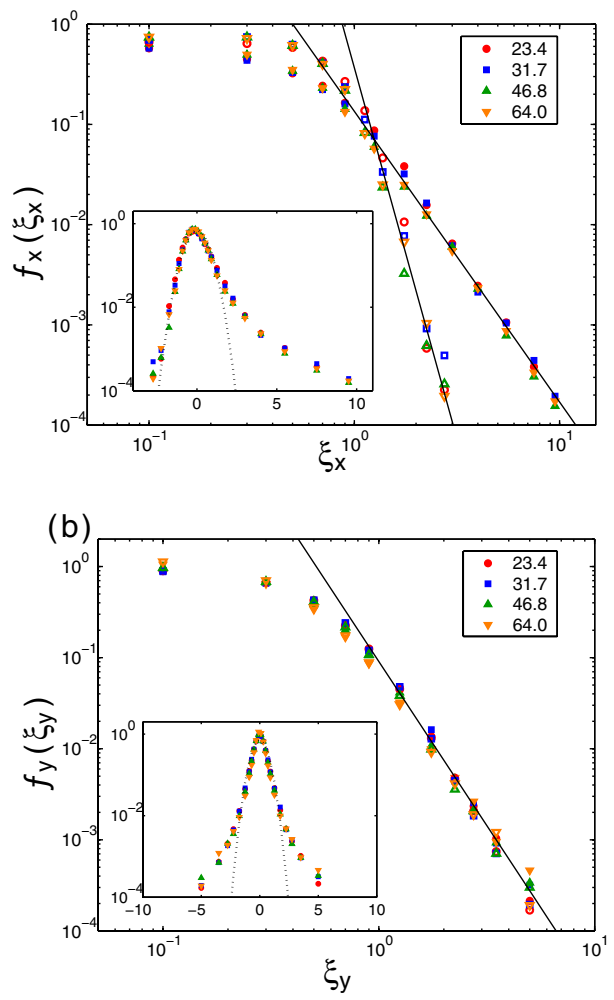

FIG. 2 (color online). Probability distribution function for (a) vertical and (b) horizontal particle velocity in the fluidlike shear layer. The data are for four channel widths with rough walls (see text), the legend giving the ratio $W / d_{p}$. The unfilled symbols in the main panels represent data for negative velocity fluctuations, reflected onto the positive side for accommodation in the log-log plot; the filled symbols represent data for positive velocity fluctuations. The inset in each panel shows the distribution in linear-log axes, the dotted line representing the Gaussian distribution of the same variance. The straight lines in the main panels are power-law fits for large velocity magnitude. ments were made far enough from the entry and exit so that the flow was fully developed; i.e., the velocity field did not vary in the direction of flow.

We note that the influence of the front glass plate on the motion of the particles complicates the interpretation of our observations. This is generally true of all imaging studies of bounded granular flows $[3,13,14]$, with the exception of a few $[4,15,16]$ that have used MRI for penetrative noninvasive imaging. Our finding that the statistical properties of the particle velocity in the core and the shear layer (defined below) are virtually identical, though the influence of the side wall is much greater in the shear layer, is perhaps an indication that our results also apply to regions far from the glass plate. However, measurement of the statistics in regions far from the boundaries by noninvasive imaging is necessary to resolve this issue.

The profiles of mean properties were determined by notionally dividing the channel into vertical bins of width $d_{p}$ and averaging the relevant particle properties in each bin over the entire duration of the video movie. Figure 1(c) shows a typical result for the mean velocity $u_{x}$ and the rootmean-square (rms) velocity fluctuation $v$. The mean velocity increases sharply within the shear layer, from a small but finite slip at the wall to the maximum value $u_{0}$, and remains constant at $u_{0}$ in the substantial core. This feature of shear banding is in agreement with previous work on
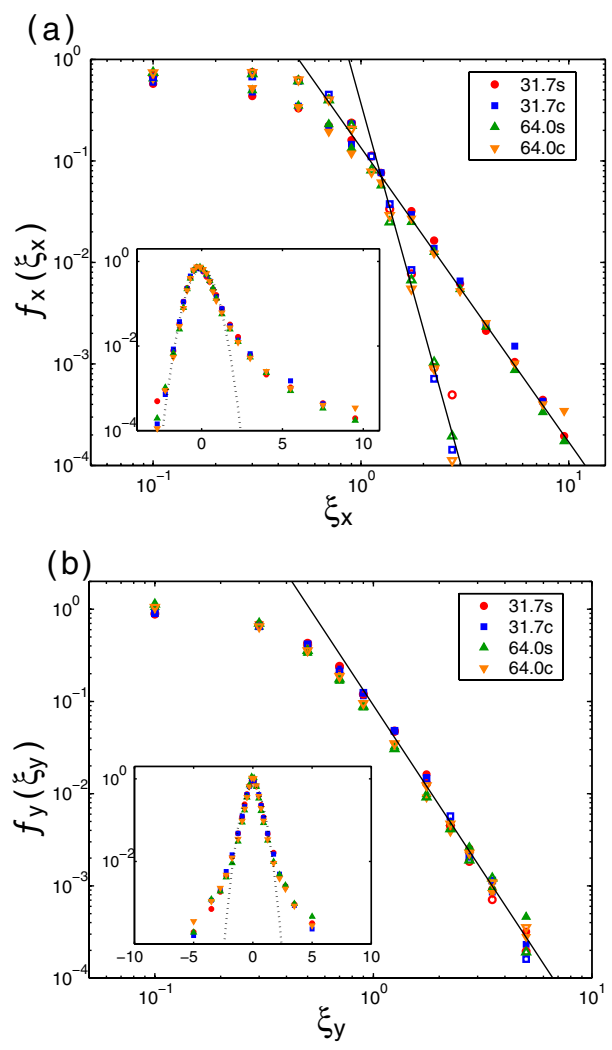

FIG. 3 (color online). Comparison of the probability distribution function in the shear layer with that in the core. The layout and symbols are as in Fig. 2. The suffixes "s" and "c" in the legend indicate data for the shear layer and the core, respectively. 
flow in channels $[3,14]$ and cylindrical Couette cells $[4,5]$. The dependence of the shear layer thickness on $W$ and the material and wall properties, and a comparison of the observed mean velocity field with predictions of available hydrodynamic theories will be reported elsewhere [17]. The conversion of energy from mean to fluctuational motion is normally high where the shear rate is high, but here we find $v$ to reach a maximum in the core, where the shear rate is negligibly small. Fluctuations in the core can arise from shear in the direction perpendicular to the glass plate, but this shear rate is estimated to be substantially lower than that in the shear layer [18]. Below we have conjectured an alternative mechanism for the generation of fluctuations in the core.

The distribution of velocities in the $x$ and $y$ directions [vertical and horizontal directions, respectively; see Fig. 1(a)] was determined as a function of position in the channel for a range of the channel width, with rough and smooth side walls. Our visualization technique could not resolve movement in the $z$ direction. The probability distribution function $f_{x}\left(\xi_{x}\right)$, where $\xi_{x} \equiv\left(c_{x}-u_{x}\right) / v$ is the dimensionless velocity fluctuation in the vertical direction, was determined by making a histogram of the number

\section{(a)}

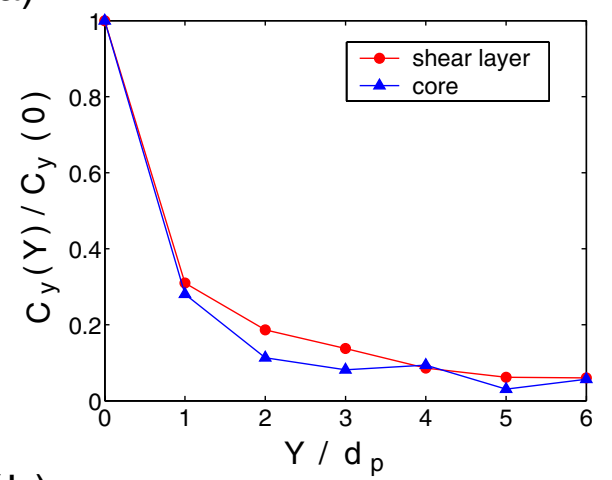

(b)

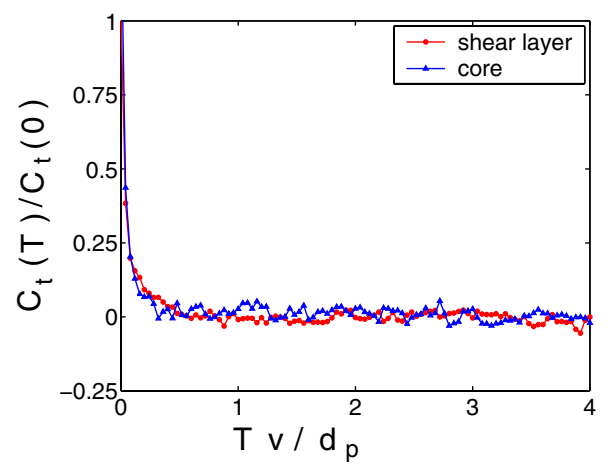

FIG. 4 (color online). Correlation functions of the velocity fluctuation in the shear layer and the core (see text for definitions of $C_{y}$ and $C_{t}$ ) for a rough walled channel with $W / d_{p}=31.7$. The reference point $y$ was $4 d_{p}$ from the left wall for the shear layer, and channel center for the core. (a) The spatial correlation as a function of the horizontal separation $Y$, and (b) the time correlation as a function of the time delay $T$ (scaled by $\left.d_{p} / v\right)$. distribution of $\xi_{x}$, and normalizing it so that $\int f_{x}\left(\xi_{x}\right) d \xi_{x}=1$; the distribution function $f_{y}\left(\xi_{y}\right)$ for horizontal velocities was determined similarly. Figure 2 shows the distribution function in the shear layer for rough walled channels of four different channel widths. We note that $f_{x}$ is not symmetric about $\xi_{x}=0$ [Fig. 2(a)], as gravity induces a preference for downward velocities. Its decay is more rapid for negative $\xi_{x}$ than for positive. There is no preferred direction for horizontal velocities and the distribution is therefore symmetric about $\xi_{y}=0$ [Fig. 2(b)]. In both directions, the distribution function decays as a power law, $f_{i}\left(\xi_{i}\right) \sim\left|\xi_{i}\right|^{-n}$, when $\left|\xi_{i}\right|$ is sufficiently large.

A striking feature of Fig. 2 is the collapse of the data for all the channel widths into a single curve. We find that data at other locations in the shear layer and for smooth walls (not shown) also collapse onto the same curve. As the flow rate, and therefore the shear rate, varied between all these experiments, this implies that $f_{x}$ and $f_{y}$ do not depend on the shear rate, but only on the local value of $v$. This result is further reinforced when we compare the data in the shear layer and the core (Fig. 3); the distribution function is identical in the two regions. This is a remarkable result, as the nature of the flow in the two regions is very different-there is continuous deformation in the shear layer, but none in the core. Curiously, this is similar to molecular fluids at thermodynamic equilibrium, where the Gaussian distribution holds regardless of the state of aggregation. However, the distribution function away from equilibrium in molecular fluids depends on gradients of the velocity and temperature [19]. Granular flows are fundamentally far from equilibrium (indeed, the flowing state bears no resemblance to the equilibrium, static, state), and hence the shear-rate independence is a significant deviation from the statistical behavior of classical fluids.

Our results are consistent with those of Choi et al. [13], who observed anisotropy and non-Gaussian distribution of the particle displacement over a time scale of $1 \mathrm{~ms}$. However, they found the distribution function changing to a Gaussian on coarse graining in time, but we find it distinctly non-Gaussian even though we measured displacements over $40 \mathrm{~ms}$. We found no perceptible change in the distribution function when a faster video camera (60 frames/s) was used [17], suggesting that our data are robust and valid over a range of time scales.

The power-law exponents were determined by fitting a straight line through the data in log-log plots for large velocities (Figs. 2 and 3). For the horizontal velocity the exponent $n_{y}$ is 3.6, and for the vertical velocity the exponents are $n_{x}^{+}=2.9$ for positive $\xi_{x}$ and $n_{x}^{-}=7.4$ for negative $\xi_{x}$. The faster decay of the distribution for negative $\xi_{x}$ is a reflection of the preference for positive (downward) velocity fluctuations that gravity induces. We do not, however, expect this form of the distribution to persist to very large velocities for the following reason: if we consider the mean velocity fluctuation square, or the specific fluctuational kinetic energy [20] $v_{\infty}^{2}=\int_{-\infty}^{\infty}\left(c_{x}-u_{x}\right)^{2} f\left(c_{x}\right) d c_{x}+$ 
$\int_{-\infty}^{\infty} c_{y}^{2} f\left(c_{y}\right) d c_{y}$, we find that the first integral is unbounded because $n_{x}^{+}<3$. Thus, for the mean kinetic energy of particles to be finite, there has to be a faster decay of $f_{x}$ for large positive $c_{x}$. This can be verified if higher particle velocities are measured, perhaps by high speed video imaging.

The presence of velocity fluctuations in the absence of shear in dense granular flows has also been observed in earlier studies, in the vicinity of a transparent wall [13], and also deep inside the flowing material [12]. A plausible explanation for this becomes apparent if we consider the spatial correlation of the velocity fluctuation, defined as $C_{y}(Y) \equiv\left\langle\bar{c}_{x}^{\prime}(t, y) \bar{c}_{x}^{\prime}(t, y+Y)\right\rangle$. Here $\bar{c}_{x}^{\prime}(t, y)$ is the instantaneous fluctuation velocity averaged over particles in the bin centered at the reference point $y, \bar{c}_{x}^{\prime}(t, y+Y)$ is the same in the bin centered at $y+Y$, and the angle brackets indicate an average over time. Figure 4(a) shows that $C_{y}(Y)$ decays rapidly with $Y$, in the shear layer and the core, but not to zero. There is a finite correlation at large distances, implying a correlated solidlike motion of the particles superimposed over the uncorrelated fluidlike motion. We believe that this solidlike motion is a result of the stick-slip [21,22], or jamming-unjamming, phenomena in granular materials. When the medium is released, or unjammed, during the slip phase, the unbalanced force accelerates the particles for a short period of time, and a part of the kinetic energy acquired by the particles is retained in fluctuational modes. The energy is lost in the next stick or jammed phase, but the cycle is repeated regularly. The decay of the time correlation, $C_{t}(T) \equiv\left\langle\bar{c}_{x}^{\prime}(t, y) \bar{c}_{x}^{\prime}(t+T, y)\right\rangle$, to zero in Fig. 4(b) indicates that the stick-slip motion itself is uncorrelated beyond a time scale of roughly half a second. [Note that $C_{t}(T)$ is not the usual autocorrelation function, as we are not tracking the velocity of individual particles in time.] The validity of this mechanism can be tested only by imaging the flow far from the boundaries noninvasively, which will also provide a clearer picture of particle dynamics in the bulk.

The above discussion leads to the important question of whether the velocity distribution we have reported is universal for slow granular flows or is limited to flows driven by a body force. This is an issue of considerable importance, and can be answered only by conducting experiments in other geometries. A kinematic variable that we have not measured in this study is the rotation velocity of the particles. Recent studies that have modeled granular media as Cosserat continua $[23,24]$ have shown the rotation velocity to play an important role in determining the kinematics. Though its measurement is more difficult, it will be useful in critically evaluating hydrodynamic theories [23-25] and in understanding the microscopic difference between the solidlike and fluidlike regions.

We thank S. Ramaswamy and N. Menon for useful discussions. This work was supported by the Department of Science and Technology, India.
*Present address: Tata Consultancy Services, Chennai, India.

[1] A.N. Schofield and C.P. Wroth, Critical State Soil Mechanics (McGraw-Hill, London, 1968).

[2] R. Jackson, J. Rheol. (N.Y.) 30, 907 (1986).

[3] R. M. Nedderman and C. Laohakul, Powder Technol. 25, 91 (1980).

[4] D. M. Mueth, G. F. Debregeas, G. S. Karczmar, P. J. Eng, S. R. Nagel, and H. M. Jaeger, Nature (London) 406, 385 (2000).

[5] W. Losert, L. Bocquet, T. C. Lubensky, and J. P. Gollub, Phys. Rev. Lett. 85, 1428 (2000).

[6] P. Dantu, in Proceedings of the Fourth International Conference on Soil Mechanics and Foundations Engineering, London (Butterworths, London, 1957), pp. 144-148.

[7] T. Wakabayashi, in Proceedings of the Ninth Japan National Congress on Applied Mechanics (Science Council of Japan, Tokyo, 1959), pp. 133-140.

[8] D. W. Howell, R. P. Behringer, and C. T. Veje, Chaos 9 , 559 (1999).

[9] F. Radjai, M. Jean, J.-J. Moreau, and S. Roux, Phys. Rev. Lett. 77, 274 (1996)

[10] F. Radjai, S. Roux, and J.-J. Moreau, Chaos 9, 544 (1999).

[11] S. G. Bardenhagen, J. U. Brackbill, and D. Sulsky, Phys. Rev. E 62, 3882 (2000).

[12] N. Menon and D. J. Durian, Science 275, 1920 (1997).

[13] J. Choi, A. Kudrolli, R. R. Rosales, and M.Z. Bazant, Phys. Rev. Lett. 92, 174301 (2004).

[14] V. V. R. Natarajan, M. L. Hunt, and E. D. Taylor, J. Fluid Mech. 304, 1 (1995).

[15] A. Caprihan and J. D. Seymour, J. Magn. Reson. 144, 96 (2000).

[16] C. Huan, X. Yang, D. Candela, R. W. Mair, and R. L. Walsworth, Phys. Rev. E 69, 041302 (2004).

[17] S. Moka and P. R. Nott (to be published).

[18] The flow rate measured at the channel exit, by weighing the material collected for a fixed period of time, was roughly $20 \%$ higher than that calculated from the velocity profile and the bulk density of a static column. The estimated velocity at the channel center then is $\approx 1.2 u_{0}$, and hence the shear rate at the core is $\approx 1 / 5$ of that in the shear layer.

[19] S. Chapman and T. G. Cowling, The Mathematical Theory of Nonuniform Gases (Cambridge University Press, Cambridge, 1964).

[20] In writing this form for $v_{\infty}$, we have assumed that the $x$ and $y$ velocities are uncorrelated. This has been verified for velocities around the mean, but the data for large velocity fluctuations are too sparse to allow verification.

[21] T. J. Budny, Powder Technol. 23, 197 (1979).

[22] S. Nasuno, A. Kudrolli, A. Bak, and J. P. Gollub, Phys. Rev. E 58, 2161 (1998).

[23] H. B. Mühlhaus and I. Vardoulakis, Géotechnique 37, 271 (1987).

[24] L. S. Mohan, P. R. Nott, and K. K. Rao, J. Fluid Mech. 457, 377 (2002).

[25] S. B. Savage, J. Fluid Mech. 377, 1 (1998). 